\title{
La Section de Sociologie de l'IFAN
}

LA section de sociologie de l'IFAN a été créée à la fin de 1952 par P. Mercier, sociologue. Elle comprend au $\mathrm{I}^{\mathrm{er}} \mathrm{J}$ anvier 1962 deux chercheurs en sociologie industrielle et psychologie sociale: A. Hauser et A. Diop, et deux chercheurs relevant d'autres disciplines: L. Massé, santé publique et $\mathrm{Y}$. Mersadier, économie.

$\mathrm{La}$ Commission de Coopération Technique en Afrique au Sud du Sahara ayant demandé aux Gouvernements membres une contribution à un projet d'investigation sur l'absentéisme et la mobilité de la main-d'œuvre, A. Hauser a été chargé par le Département, en 1959, d'une étude de ces variables dans la région de Dakar. Une enquête portant sur une période d'un an a été effectuée auprès de 2.600 manœuvres, ouvriers et agents de maîtrise de huit entreprises choisies dans les industries de transformation et les ateliers de mécanique générale; une enquête complémentaire a été effectuée auprès de 447 de ces travailleurs, à l'aide d'un questionnaire d'attitudes envers le travail industriel.

En 1959 et 1960 ont été effectuées par Y. Mersadier deux enquêtes. L'une en Mauritanie, dans les centres principaux, à la demande du gouvernement de cet État, ne concerne que les manœuvres. Ils ont été étudiés dans quatre principaux centres: Nouakchott, Rosso, PortÉtienne et Kaëdi. Des relevés de prix ont été effectués dans les autres centres.

A Dakar, une enquête a débuté en Mars 1960 à Juillet 196r. Elle a porté sur 900 familles tirées aléatoirement; un échantillon principal d'environ 4.000 familles a été préalablement constitué par inventaire de $\mathrm{I} / \mathrm{ro}$ des concessions de la ville. Cette dernière enquête, qui s'est déroulée avec des crédits du FAC, a été effectuée en collaboration avec le service de la statistique du Gouvernement du Sénégal. Le dépouillement en sera terminé durant le deuxième trimestre de 1962 .

A partir du même échantillon principal, Y. Mersadier a effectué une enquête sur la structure du chômage à Dakar et les possibilités de retour à la campagne des chômeurs. Les résultats paraîtront également au cours du $2^{e}$ trimestre de 1962 .

A. Diop a effectué une étude sur l'immigration des Toucouleurs à Dakar, pendant 9 mois, de Juillet I958 à Mars I959. L'enquête sur le terrain effectuée avec le procédé du sondage aléatoire a touché plus de 600 sujets sur une population immigrée estimé à près de 25.000 , ce qui donne une précision satisfaisante. Les résultats ont fait l'objet d'un rapport.

De Juin 1960 à Janvier $196 \mathrm{r}, \mathrm{L}$. Thoré a effectué une enquête sur la ville-satellite de Dakar, Dagoudane-Pikine. Il s'agit d'abord d'obtenir une idée assez précise de la structure sociale de cette ville de création récente, ensuite d'étudier l'effet de l'urbanisation sur un point particulier: la société familiale, en tenant compte des conditions de logement.

Pour compléter cette enquête, une étude parallèle va être entreprise dans deux ou trois villages wolof de la région des Niayes, sur la structure actuelle de la famille. C'est la comparaison entre les résultats de ces deux travaux qui permettra d'apprécier l'importance des changements socio-culturels en cours, qui séparent la campagne de la ville.

\section{The Economic History of Lagos}

Mr. A. G. Hopkins, a tesearch student at the School of Oriental and African Studies, is studying the economic history of Lagos with particular reference to the part played by African merchants in the period I $85 \mathrm{I}-1920$. He has been awarded a Commonwealth Scholarship to enable him to spend the current academic year (196I-2) in Nigeria. Among other sources Mr. Hopkins hopes to be able to make use of family papers belonging to the nineteenth-century African merchants and now in the possession of their relatives. 